\title{
Looking at the Physics of What a Quark Star-Black Hole Binary Would Create in Terms of GW Signals and New Physics
}

\author{
Andrew Beckwith \\ Physics Department, College of Physics, Chongqing University, Chongqing, China \\ Email: rwill9955b@gmai.com
}

How to cite this paper: Beckwith, A. (2021) Looking at the Physics of What a Quark Star-Black Hole Binary Would Create in Terms of GW Signals and New Physics. Journal of High Energy Physics, Gravitation and Cosmology, 7, 1255-1263. https://doi.org/10.4236/jhepgc.2021.74075

Received: June 25, 2021

Accepted: August 24, 2021

Published: August 27, 2021

Copyright $\odot 2021$ by author(s) and Scientific Research Publishing Inc. This work is licensed under the Creative Commons Attribution International License (CC BY 4.0).

http://creativecommons.org/licenses/by/4.0/

\begin{abstract}
A quark star, black hole pairing as a would-be Gravitational wave generator is brought up. Quark stars are, anyway, likely to be black holes, above a certain mass limit, whereas a quark star in itself obey thermodynamic "laws" which in certain ways differ from the traditional black hole models. We list some of the probable consequences of such a binary, and make predictions as to certain GW phenomenon which will have observational consequences. i.e., a GW "change in energy" from a black hole-Quark star pair would likely be within $90 \%$ of that of comparatively massed black hole-black hole binary pair. The electromagnetic "profile" of the two cases would differ dramatically, and we conclude our inquiry with an open question if a generalized uncertainty principle could play a role in comparing the $7^{\text {th }}$ and $8^{\text {th }}$ equations of our presentation.
\end{abstract}

\section{Keywords}

GW Signals, Quark Star-Black Hole Binary, New Physics

\section{Introduction}

It is simple enough to visualize what happens if a quark star and a black hole binary exist and what this says about GW generation is open to speculation.

See this, i.e., speculation which is revealed as to what has been suggested is an actual quark star-black hole binary pair.

In addition, we also in the end of this document include what may be a linkage as to what is known as GRBs and the formation of a quark star, from a massive GRB formation explosion.

The following are from an article as to the weird physics of quark star black 
hole binary pairs.

In a sense profoundly elementary but with significant surprises in the conclusion.

- Text of what Figure 1 is referring to is

- Quote

- On the $14^{\text {th }}$ of August 2019, the LIGO-Virgo collaboration detected a gravitational wave signal believed to be associated with the merging of a binary stellar system composed of a black hole with a mass of 23 times the mass of the sun $\left(M_{\odot}\right)$ and a compact object with a mass of about $2.6 M_{\odot}$. The nature of GW190814's secondary star is enigmatic, since, according to the current astronomical observations, it could be the heaviest neutron star, or the lightest black hole ever observed

- End of quote

- From [2] we have the following quote

- Quote

- NSs and QSs could coexist, as has been proposed and discussed in detail in several papers

- End of quote

- i.e., one of the models proposed is, in [3] making a pitch for a sudden release of energy as to the following mechanism

- Quote

- The total energy released in the NS $\rightarrow$ SS conversion is given by the difference between the total binding energy of the strange star $\mathrm{BE}(\mathrm{SS})$ and the total binding energy of the neutron star $\mathrm{BE}(\mathrm{NS})$

- End of quote

- In so many words, the template for examining what may be happening, hinges upon having a bust of energy, and a difference of energy between a neutron star and a strange star being released. We see though that this does NOT have a lot to do with how to observe a change in Gravitational energy as given in the following

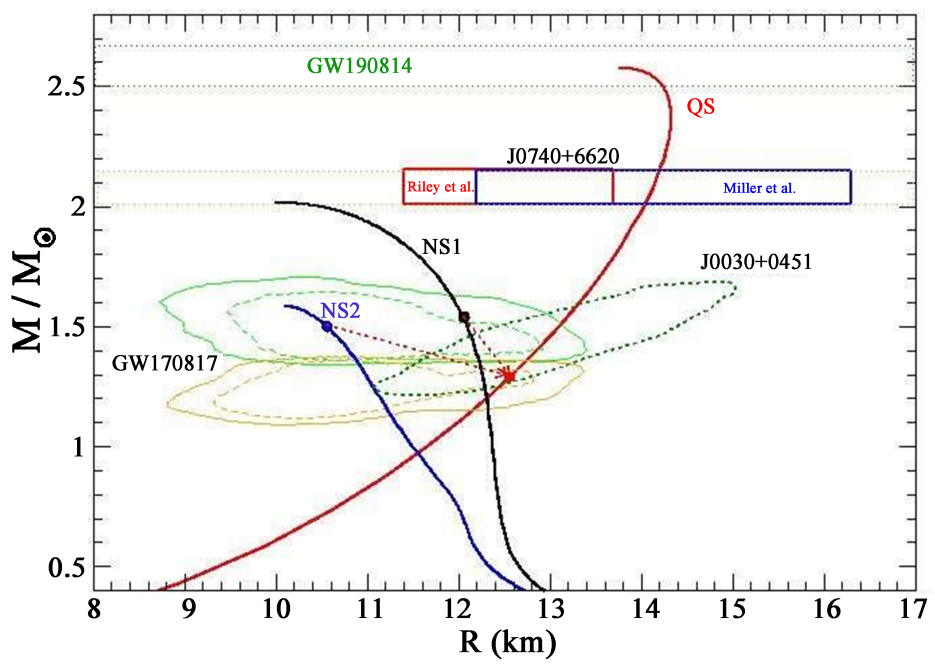

Figure 1. From [1]. 
What does this have to do with a quark star-black hole binary? Simply put, look at Maggiore, Volume 1 [4] as to what is stated if one has a change in energy, on page 175 which is stated as

$$
\begin{aligned}
& \Delta E_{\text {Gravitational radiation binaries }} \approx 8 \times 10^{-2} \tilde{\mu} c^{2} . \\
& \tilde{\mu} \equiv \text { reduced mass of system }
\end{aligned}
$$

The nub of this is that for a binary system with a black hole, and quark star that the value of Equation (1) would be enormous, whereas we can do a further refinement by writing the reduced mass as

$$
\tilde{\mu}=\frac{m_{1} m_{2}}{m_{1}+m_{2}} .
$$

And then from [4] we would be observing a binding energy given as with respect to a Schwarzschild metric [4] [5].

$$
E_{\text {Gravitational radiation BINDING ENERGY }} \approx 5.7 \times 10^{-2} \tilde{\mu} c^{2}
$$

In doing so, we have to consider what happens to the reduced mass, if we have a transfer from say a Neutron star to a quark star, as one of the constituent components of the reduced mass [6] [7] [8] [9].

\section{Neutron Star to Quark Star Transformation, and Its Energy Release}

The smallest, most massive, most compressed neutron star possible is about 17 kilometers in diameter. A quark star can be smaller than 11 kilometers diameter, Having said that consider, now a transfer of a neutron star to a quark star and what it entails [10].

Quote

"The possible existence of two families of compact stars, neutron stars and quark stars, naturally leads to a scenario in which a conversion process between the two stellar objects occurs with a consequent release of energy of the order of $10^{53}$ erg" [11].

End of quote

In so many words, we can have that we would have that $10^{53}$ ergs is $6.24150913 \times 10^{55}$ giga electron volts, or 6.24 times $10^{55} \mathrm{GeV}$. Hence, this transfer of energy from Neutron star to quark star would entail a gigantic pulse of energy, whereas a quark star would have about $10 \%$ less energy than a typical black hole of equal mass. Note the following.

Quote

The dust cloud surrounding black holes becomes heated by the incredible gravitational forces, which emits extremely powerful radiation. A quark star is expected to emit radiation, just $10 \%$ less powerful.

End of quote 
i.e., the final stop as far as deciding. i.e., a GRB could in fact be signaling a transfer from a neutron star to quark star [10] [11], whereas [12] specifically refers to accreditation disks of a quark star as different from a black hole.

For a black hole, note the following [12]

In the Biermann battery mechanism for B field creation about a black hole, the mere act of electric charges in an accreditation disk about a black hole will create magnetic fields, and this has been understood since 1975 . We likely would still, by [12] see an accreditation disk about a quark star, with, also, B field generation.

From [12]

A. However, strange stars exhibit a low-luminosity but high-temperature bremsstrahlung spectrum, which, in combination with the emission properties of the accretion disc, may be the key signature to differentiate massive, strange stars from the black hole.

B. The punch line shows up on page 12 of [13].

Quote

"The characteristics of the disk spectra around black holes and quark stars also exhibit significant differences. For black holes, the maxima of the spectra are located at higher frequencies and reach higher values than those for quark stars. In case of the static CFL quark stars with the maximal total mass, both the spectral amplitude, and the frequency at which the maximum is located, have lower values than those for the rotating cases (with higher maximal masses)".

i.e., see this, below, this is our Figure 2. i.e., CFL would be for a quark star whereas Kerr BH would be for a Kerr black hole.

The temperature profiles of the thin accretion disk around rotating black holes and quark stars with the same total mass $\mathrm{M}$ and spin parameter a. The static configuration is also presented.

End of quote

This is for electromagnetic generation of $\mathrm{E}$ and $\mathrm{M}$ signals from a quark star versus what we would see for a straight-out black hole would be extremely different.

On the other hand, the existence of a gamma ray burst, and a gravitational wave source would still have co existing properties as seen below Figure 3 .

Likely, the GW signal created between a black hole, black hole binary, would obey Equation (2) and be ALMOST duplicated by a black hole, Quark Star. As in Figure 2 though, for [12], the differences would show up most decisively in Magnetic field behavior between the Quark star and black holes. i.e., electromagnetics and the temperature mappings of the accreditation disks would be very different.

The companion star would likely be a quark star, with an accreditation disk being siphoned off to feed the black hole as is show in Figure 4, with the situation described as in [14]. 

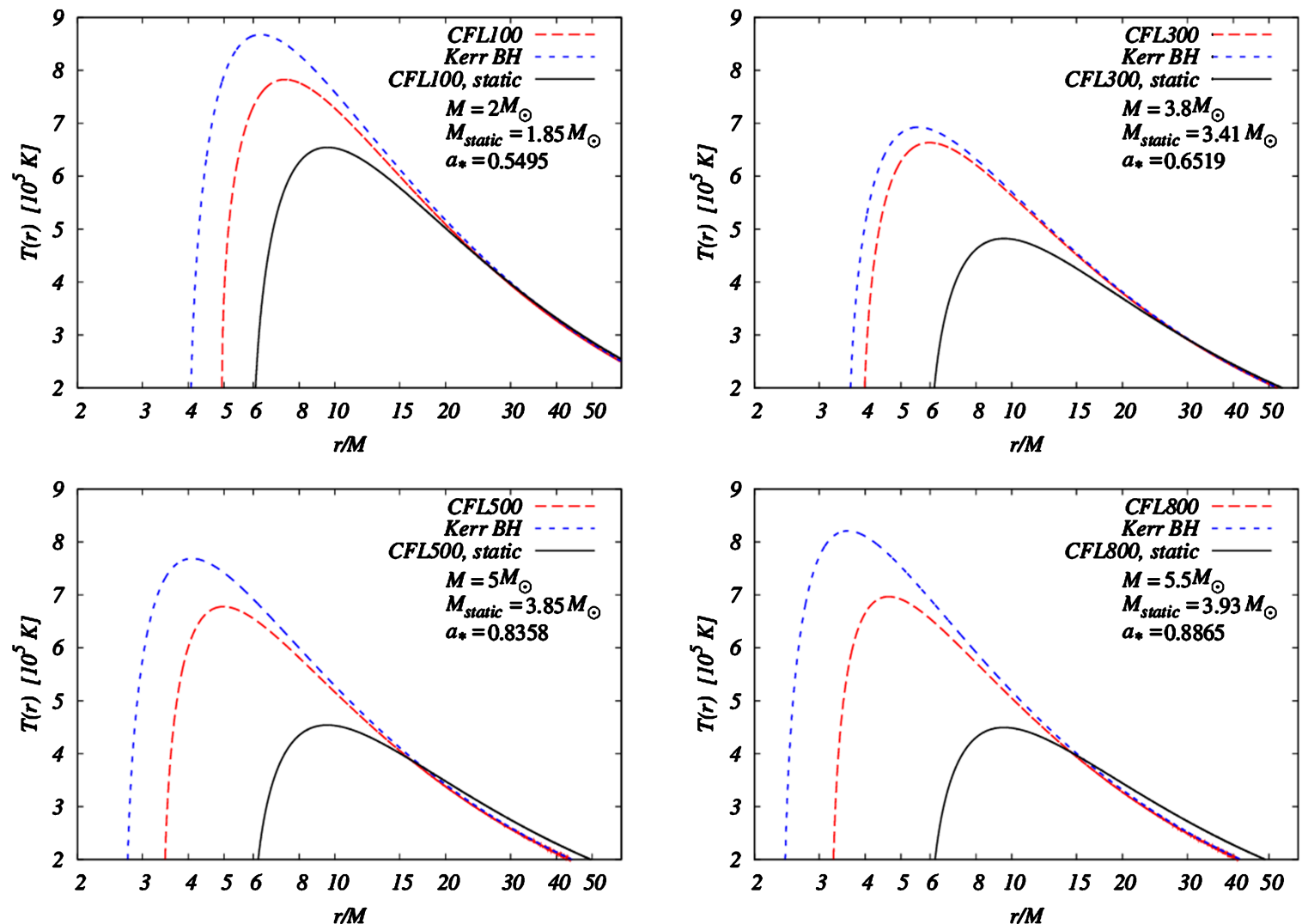

Figure 2. From [12] where we have that as to the results from [12] and its Figure 4 quote.

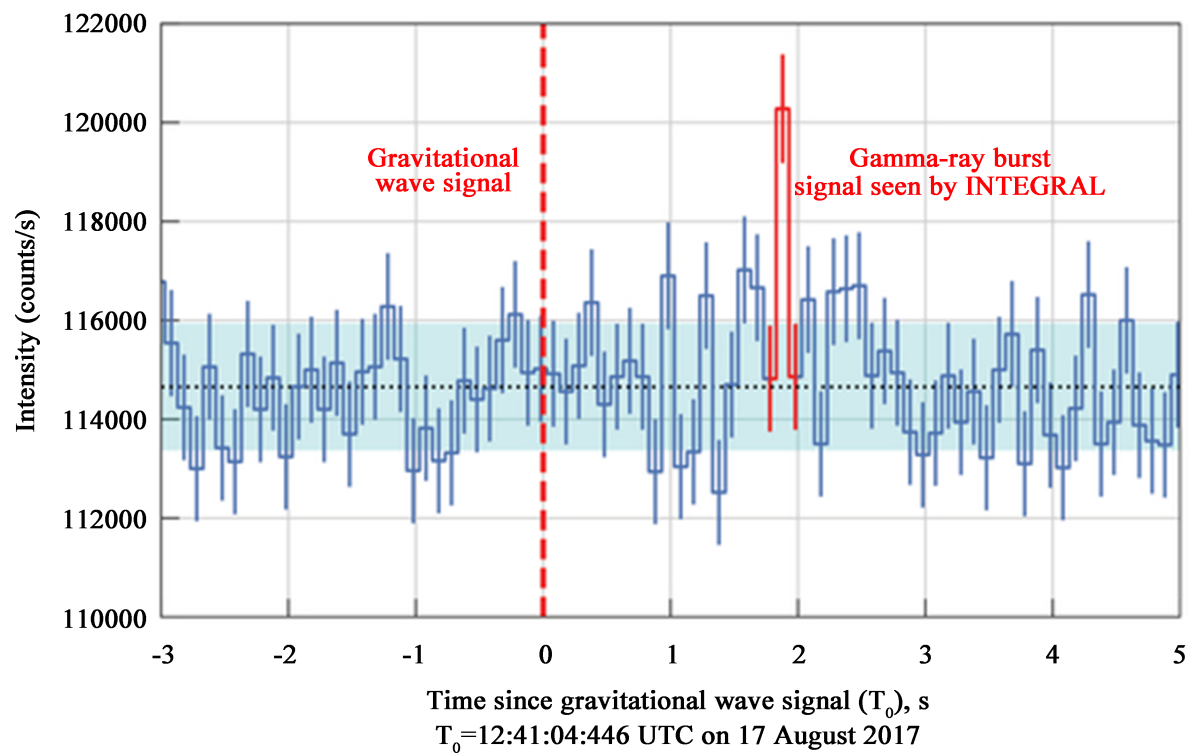

Figure 3. from [13] states that there would be close synchronization of time between a gravitational wave signal, and gamma ray bursts, in an event likely connected to a quark star being formed.

Figure 4 is what would happen, if we were looking at the physics of [1] [2] [3], and it leads to the following question to ask, i.e., how can we combine the results of both a GRB burst and a quark star-black hole? 


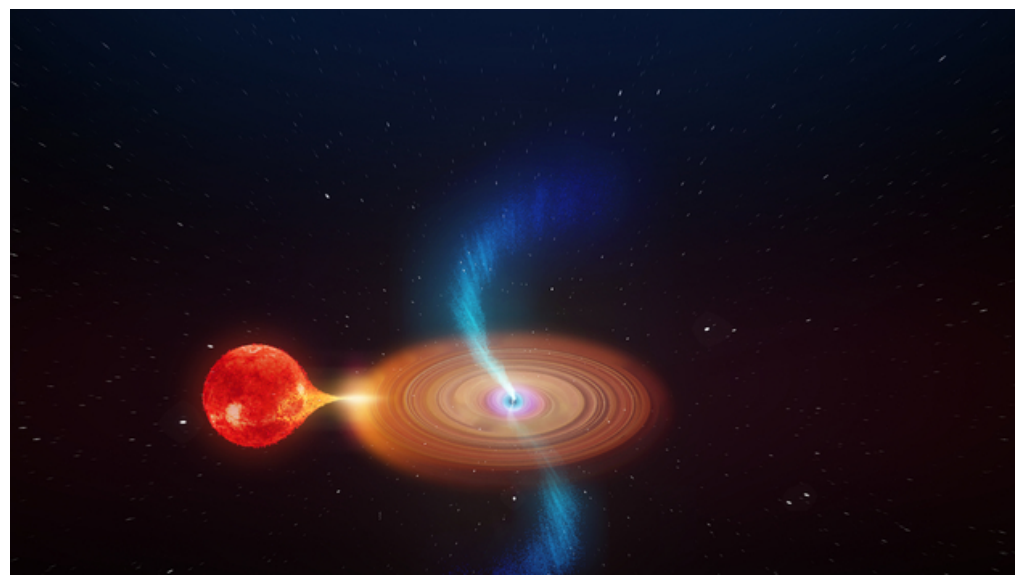

Figure 4. From [14], i.e. Artist's impression of a black hole accreting material from a companion star.

To do this, consider [15] which has them a reference as to what is called of black hole mining. To do this use from [15] the so-called black hole mining in a black hole atmosphere argument to have a high temperature, Low Luminosity behavior of a quark star.

i.e., on page 340 ,

$$
\Delta E_{\text {mined }} \approx \frac{4}{3} \cdot \breve{a} G_{\breve{a}} \cdot \frac{T_{H}^{4}}{\breve{\alpha}^{3}} \cdot V .
$$

Here, will refer to a so-called atmosphere's law of blueshift, [16].

$$
T=\left(T_{H} / \breve{\alpha}\right)=\text { atmosphere law of blueshif } t
$$

The precise language is that a quark star has a very temperature value and low Luminosity. i.e. which could be obtained, in [16], page 16, via

$$
L=\operatorname{Luminosity}(\text { Quark Star })=\sigma T_{H}^{4}\left(R_{e f f}\right) \times 4 \pi R_{e f f}^{2} .
$$

Whereas we have for Equation (5) being low, that $T_{H}$ is much smaller than Planck temperature, whereas Equation (4) will be comparatively enormous for a quark star, due to the smallness of a picked $\breve{\alpha}$ term, the Equation (5) term would be comparatively enormous. Hence, to first order, due to the specifics of the quark star being formed, we could state.

$$
\Delta E_{\text {mined }} \approx \frac{4}{3} \cdot \breve{a} G_{\breve{a}} \cdot \frac{T_{H}^{4}}{\breve{\alpha}^{3}} \cdot V \propto 6.24 \times 10^{55} \mathrm{GeV}
$$

Note, Equation (6) is for a GRB which, initially is NOT dominated by GW.

In this case, the alleged change in "mined" energy would be enormous and related to a GRB 'signal' for an evolution from a neutron star to a quark (strange) star. Due to the smallness of a picked $\breve{\alpha}$ term, whereas we will use the following term for GW release of energy for a quark-star black hole binary.

$$
\Delta E_{\text {quark star black hole }} \approx 5.7 \times\left(\frac{m_{\text {quark star }} \cdot m_{\text {Black hole }}}{m_{\text {quark star }}+m_{\text {Black hole }}}\right) \cdot c^{2}
$$

This should be compared, Equation (7) with respect to a derivation in [16] in 
page 98 which does not include relativistic effects but is the result of a slamming of a quark star with a black hole.

Note that in the below, if the magnitude of Equation (7) and Equation (8) are the same, it will then.

lead to a question as to how to interpret the final period of rotation of a quark star with a black hole, before merger of the two masses, which we call $\tilde{T}_{1}^{2 / 3}$ which the final period of rotation between the two masses prior to ringdown merger of the two, to the $2 / 3^{\text {rd }}$ power.

$$
\begin{aligned}
& -\Delta E(\text { quark star BH merge }) \\
& \approx \frac{(2 \pi G)^{2 / 3} \cdot\left(m_{\text {quark star }}+m_{\text {Black hole }}\right)^{2 / 3}}{2 \tilde{T}_{1}^{2 / 3}} \cdot\left(\frac{m_{\text {quark star }} \cdot m_{\text {Black hole }}}{m_{\text {quark star }}+m_{\text {Black hole }}}\right)
\end{aligned}
$$

\section{Conclusion and Future Question to Ask, Which Is Significant}

Figure 3, Figure 4, and Equations (6)-(8) need to be numerically simulated. But the real question is, after the GRB blow up would as an example there be an accreditation disk as show with regards, say to a quark star. The models indicate yes, but we do not know even now the precise nature of the electromagnetic signals, or how long they would be lasting,

As an example, the Bierman battery model has been proposed to give a tentative magnetic field, in tandem with GW releases, as specified. What is that $\mathrm{B}$ field detail, as compared to the final Period of rotation, as specified in Equation (8)? We still do not know. In addition, Equation (7) and Equation (8) assume that we have special relativity applied? Could as an example, quantum effects enter in, as say in a GOP (Generalized Uncertainty principle) as specified in [17], whereas also [18] and [19] have further issues awaiting review.

Our final comment needs to be investigated. See this from [20], namely the issue of Bose Einstein condensation w.r.t Gravitons as a condensate for the formation of black holes, and does this play a role in our binary of a quark starblack hole model?

We think it does, in a major way. Here is the idea, i.e., that Black holes, as Bose Einstein condensates of gravitons, will naturally interact with respect to the physics of Equation (4), Equation (7) and Equation (8) and are essential to theoretical justifications of our inquiry.

$$
\begin{aligned}
& m \approx \frac{M_{P}}{\sqrt{N_{\text {gravitons }}}} \\
& M_{B H} \approx \sqrt{N_{\text {gravitons }}} \cdot M_{P} \\
& R_{B H} \approx \sqrt{N_{\text {gravitons }}} \cdot l_{P} \\
& S_{B H} \approx k_{B} \cdot N_{\text {gravitons }} \\
& T_{B H} \approx \frac{T_{P}}{\sqrt{N_{\text {gravitons }}}}
\end{aligned}
$$


Here, the first term, $\mathrm{m}$, is in the effective mass of a graviton. This is my take as to how to make all this commensurate as to special relativity.

$$
\begin{gathered}
m \approx \frac{m_{g}}{\sqrt{1-\left(\frac{v_{g}}{c}\right)^{2}}} \approx \frac{M_{P}}{\sqrt{N_{\text {gravitons }}}} \approx 10^{-10} \text { grams } \\
\therefore N_{\text {gravitons }} \approx 10^{10}
\end{gathered}
$$

Do Equation (9), Equation (10) and Equation (11) dovetail in our investigation of Equation (4), Equation (7) and Equation (8)? We believe the answer is yes, and that we need to understand this fully in order to answer foundational questions.

\section{Conflicts of Interest}

The author declares no conflicts of interest regarding the publication of this paper.

\section{References}

[1] Fadelli, I. (2021) Could the Source of the GW190814 Event be a Black Hole-Strange Quark Star System? https://phys.org/news/2021-06-source-gw190814-event-black-hole-strange.html

[2] Bombaci, I., Drago, A., Logoteta, D., Pagliara, G. and Vidaña, I. (2021) Was GW190814 a Black Hole-Strange Quark Star System? Physical Review Letters, 126, Article ID: 162702. https://arxiv.org/pdf/2010.01509.pdf https://doi.org/10.1103/PhysRevLett.126.162702

[3] Bombaci, I. and Datta, B. (2000) Conversion of Neutron Stars to Strange Stars as the Central Engine of Gamma-Ray Bursts. The Astrophysical Journal, 530, L69-L72. https://doi.org/10.1086/312497

[4] Maggiore, M. (2008) Gravitational Waves: Vol. 1: Theory and Experiments. Oxford University Press, New York City.

[5] Landau, L.D. and Lifshitz, E.M. (1979) Course of Theoretical Physics Vol. 2: The Classical Theory of Fields. 4th Revised Edition, Pergamon, Oxford University Press, Oxford, UK.

[6] Berezhiani, Z., Bombaci, I., Drago, A., Frontera, F. and Lavagno, A. (2003) Gamma Ray Bursts from Delayed Collapse of Neutron Stars to Quark Matter Stars. The Astrophysical Journal, 586, 1250-1253. https://doi.org/10.1086/367756

[7] Bombaci, I., Parenti, I. and Vidaña, I. (2004) Quark Deconfinement and Implications for the Radius and the Limiting Mass of Compact Stars. The Astrophysical Journal, 614, 314-325. astro-ph/0402404. https://doi.org/10.1086/423658

[8] Drago, A., Lavagno, A. and Pagliara, G. (2004) Effects of Color Superconductivity on the Structure and Formation of Compact Stars. Physical Review D, 69, Article ID: 057505. https://doi.org/10.1103/PhysRevD.69.057505

[9] Drago, A., Lavagno, A. and Pagliara, G. (2014) Can Very Compact and Very Massive Neutron Stars Both Exist? Physical Review D, 89, Article ID: 043014. https://doi.org/10.1103/PhysRevD.89.043014

[10] Pagliara, G. (2014) On the Conversion of Neutron Stars into Quark Stars. EPJ Web of Conferences, 66, Article ID: 07018. https://doi.org/10.1051/epjconf/20146607018 
https://arxiv.org/abs/1312.1083

[11] Drago, A., Pagliara, G. and Parenti, I. (2006) Transition to Quark Matter and Long Gamma Ray Bursts. Il Nuovo Cimento B, 121, 1355-1361.

https://arxiv.org/abs/astro-ph/0701240

[12] Kovacks, Z., Cheng, K.S. and Harko, T. (2009) Can Stellar Mass Black Holes Be Quark Stars? https://arxiv.org/pdf/0908.2672.pdf

[13] European Space Agency (2017) Gamma-Ray Burst after Gravitational Waves. https://sci.esa.int/web/integral/-/59670-gamma-ray-burst-after-gravitational-waves

[14] European Space Agency (2019) Black Hole Accreting Material from Its Companion Star.

https://sci.esa.int/web/integral/-/61323-black-hole-accreting-material-from-its-com panion-star

[15] Thorne, K., Price, R. and MaCDonald, D. (1986) Black Holes, the Membrane Paradigm. Yale University Press, Yale.

[16] Weinberg, S. (2020) Lectures on Astrophysics. Cambridge University Press, Cambridge.

[17] Beckwith, A. and Moskaliuk, S. (2017) Generalized Heisenberg Uncertainty Principle in Quantum Geometrodynamics and General Relativity. Ukrainian Journal of Physics, 62, 727-740. https://doi.org/10.15407/ujpe62.08.0727 https://ujp.bitp.kiev.ua/index.php/ujp/article/view/2018650/757

[18] Maldacena, J. (2020) Comments on Magnetic Black Holes. Journal of High Energy Physics, 79. https://arxiv.org/pdf/2004.06084.pdf

[19] Thorne, K.S., Zurek, W.H. and Price, R. (1986) The Thermal Atmosphere of a Black Hole. In: Thorne, K., Price, R. and MacDonald, D., Eds., Black Holes, the Membrane Paradigm, Yale University Press, New Haven, 280-340.

[20] Chavanis, P.H. (2012) Self Gravitating Bose-Einstein Condensates. In: Calmet, X., Ed., Quantum Aspects of Black Holes, Vol. 178, Springer Nature, Cham, 151-194. https://doi.org/10.1007/978-3-319-10852-0_6 